Gilchrist, H.G., Mallory, M.L., 2005. Declines in abundance and distribution of the Ivory Gull (Pagophila eburnea) in Arctic Canada. Biological Conservation 121, 303-309.

Haney, J.C., MacDonald, S.D., 1995. Ivory Gull (Pagophila eburnea). In: Poole, A., Gill, F. (Eds.), The birds of North America, No. 175. The Academy of Natural Sciences, Philadelphia and The American Ornithologists' Union, Washington, DC, 24 p.

Hobson, K.A., 1995. Reconstructing avian diets using stable-carbon and nitrogen isotope analysis of egg components: patterns of isotopic fractionation and turnover. The Condor 97, 752-762.

Hobson, K.A., Fisk, A., Karnovsky, N., Holst, M., Gagnon, J-M., Fortier, M., 2002. A stable isotope $\left(\delta^{13} \mathrm{C}, \delta^{15} \mathrm{~N}\right)$ model for the North Water food web: implications for evaluating trophodynamics and the flow of energy and contaminants. Deep-Sea Research II 49, 51315150.

Hunt Jr., G.L., 1991. Occurrence of polar seabirds at sea in relation to prey concentrations and oceanographic factors. Polar Research 10, $553-559$.

Kelly, T.M., Jones, J.D., Smith, G.R., 1975. Historical changes in mercury contamination in Michigan walleyes (Stizostedion vitreum vitreum). Journal of the Fisheries Research Board of Canada 32, 17451754.

Macdonald, R.W., Harner, T., Fyfe, J., 2005. Recent climate change in the Arctic and its impact on contaminant pathways and interpretation of temporal trend data. The Science of the Total Environment 342, $5-86$.

Muir, D., Braune, B., DeMarch, B., Norstrom, R., Wagemann, R., Lockhart, L., Hargrave, B., Bright, D., Addison, R., Payne, J., Reimer, K., 1999. Spatial and temporal trends and effects of contaminants in the Canadian Arctic marine ecosystem: a review. The Science of the Total Environment 230, 83-144.
Nielsen, C.O., Dietz, R., 1989. Heavy metals in Greenland seabirds. Meddelelser om Grønland, Bioscience 29, 3-26.

Sarakinos, H.C., Johnson, M.L., Vander Zanden, M.J., 2002. A synthesis of tissue-preservation effects on carbon and nitrogen stable isotope signatures. Canadian Journal of Zoology 80, 381-387.

StatSoft Inc., 1994. Statistica for Windows. StatSoft Inc., Tulsa, OK.

Stern, G.A., Macdonald, R.W., 2005. Biogeographic provinces of total and methyl mercury in zooplankton and fish from the Beaufort and Chukchi seas: results from the SHEBA drift. Environmental Science and Technology 39, 4707-4713.

Thompson, D.R., 1996. Mercury in birds and terrestrial mammals. In Beyer, W.N., Heinz, G.H., Redmon-Norwood, A.W. (Eds.), Environmental Contaminants in Wildlife: Interpreting Tissue Concentrations. SETAC Special Publication Series. Lewis Publishers, Boca Raton, FL, pp. 341-356.

Thyen, S., Becker, P.H., Behmann, H., 2000. Organochlorine and mercury contamination of little terns (Sterna albifrons) breeding at the western Baltic Sea, 1978-96. Environmental Pollution 108, 225-238.

Tieszen, L.L., Boutton, T.W., Tesdahl, K.G., Slade, N.H., 1983 Fractionation and turnover of stable carbon isotopes in animal tissues: implications for $\delta^{13} \mathrm{C}$ analysis of diet. Oecologia 57, 32-37.

Wagemann, R., Trebacz, E., Boila, G., Lockhart, W.L., 1998. Methylmercury and total mercury in tissues of arctic marine mammals. The Science of the Total Environment 218, 19-31.

Wiener, J.G., Krabbenhoft, D.P., Heinz, G.H., Scheuhammer, A.M., 2003. Ecotoxicology of mercury. In: Hoffman, D.J., Rattner, B.A., Burton, G.A. Jr., Cairns, J. Jr. (Eds.), second ed., Handbook of Ecotoxicology Lewis Publishers, Boca Raton, FL, pp. 409-463.

Wolfe, M.F., Schwarzbach, S., Sulaiman, R.A., 1998. Effects of mercury on wildlife: a comprehensive review. Environmental Toxicology and Chemistry 17, 146-160.

\title{
Trace metal contamination and benthic assemblages in Subaé estuarine system, Brazil
}

\author{
V. Hatje ${ }^{a, *}$, F. Barros ${ }^{b}$, D.G. Figueiredo ${ }^{a}$, V.L.C.S. Santos ${ }^{a}$, M.C. Peso-Aguiar ${ }^{b}$ \\ a Departamento de Química Analítica, Instituto de Química, Universidade Federal da Bahia, Campus Ondina, Salvador, BA 40170-290, Brazil \\ b Departamento Zoologia, Instituto de Biologia, Universidade Federal da Bahia, Campus Ondina, Salvador, BA 40170-290, Brazil
}

The Subae is one of the main tributaries of the Todos os Santos Bay (BTS), the second largest bay in Brazil, with an area of approximately $1100 \mathrm{~km}^{2}$. Subaé has a catchment area of $390 \mathrm{~km}^{2}$, is $50 \mathrm{~km}$ long, and discharges are modest $\left(4.5 \mathrm{~m}^{3} / \mathrm{s}\right)$. Nevertheless, it is the second most important source of freshwater and suspended material to the BTS (CRA, 2004). Estuarine conditions are found downstream of Santo Amaro Municipality (Fig. 1). The tides are semi-diurnal, with a mean spring tide of $2.0 \mathrm{~m}$.

It has been reported that a primary lead smelter that operated between 1960 and 1993 in the Subaé catchment, in Santo Amaro, has released large amounts of metals,

\footnotetext{
* Corresponding author. Tel./fax: +557132355166

E-mail address: vhatje@ufba.br (V. Hatje).
}

especially $\mathrm{Pb}$ and $\mathrm{Cd}$. The $\mathrm{Pb}$ smelter produced $11-$ $32 \times 10^{6} \mathrm{~kg}$ of $\mathrm{Pb}$ bars/year. It is estimated that, during its operation, more than $25 \times 10^{7} \mathrm{~kg}$ of $\mathrm{Cd}$ were discharged in the river, and at least another $15 \times 10^{7} \mathrm{~kg}$ have been released in the atmosphere. The lead burden is difficult to estimate. Until 1980 the smelter dross (1-3\% lead) was freely given to the population for paving yards and was used extensively by the local council for paving streets and public places, including local schools (Silvany-Neto et al., 1989).

The smelter has given rise to many health-related complaints from local residents concerning domestic animals and crops. Over the years, it has also been found that the human population, especially children, was heavily contaminated (Carvalho et al., 1984, 1985, 1989, 2003). Reis 


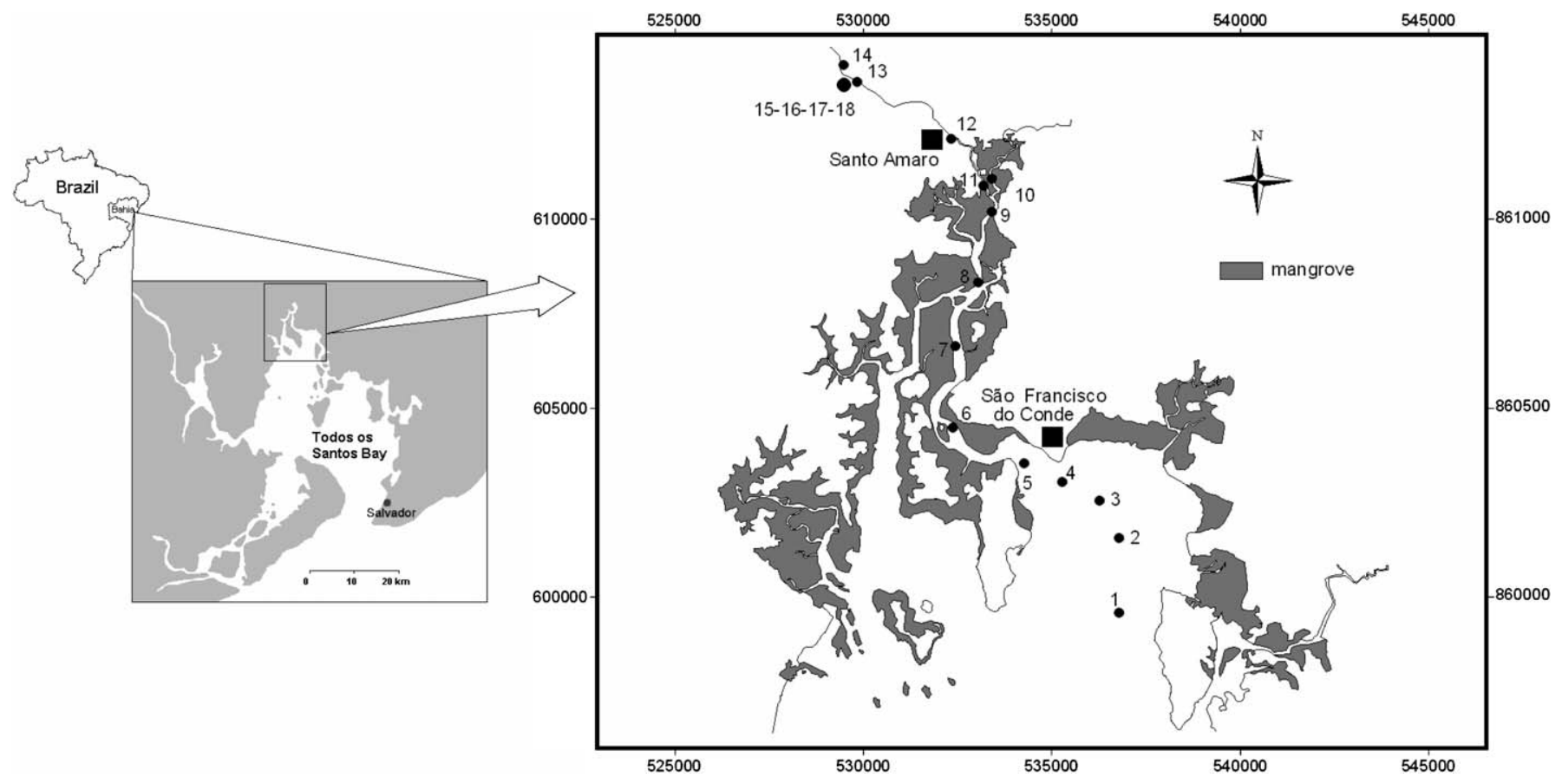

Fig. 1. Sampling location in Subaé system, Bahia, Brazil (estuarine samples: \#1-11; river samples: \#12-14; soil samples: \#15-18).

(1975) showed that $\mathrm{Cd}$ and $\mathrm{Pb}$ concentrations in the waters of Subaé exceeded the World Health Organization tolerance limits by 8 and 16 times, respectively. High concentrations of trace metals in sediments and mollusks collected from Subaé estuary have also been reported (Tavares, 1996; CRA, 2004).

It is now more than ten years since the smelter was active. However, around $50 \times 10^{7} \mathrm{~kg}$ of metal enriched wastes are stored in the yards of the old smelter. This waste disposal site is open to the elements and is located no more than $500 \mathrm{~m}$ away from the river. The amount of contaminated material removed by surface run-off and ground water to the Subae estuarine system and BTS has yet to be determined. In addition, there are two fishing villages and other important industrial activities in the basin, including a paper mill, sugar, and chemical industries.

Few studies have been undertaken in the area since the 1990s (Tavares, 1996; Carvalho et al., 2003; CRA, 2004). These studies had focused in the vicinity of the smelter in Santo Amaro Municipality, and no information has been published in the scientific literature assessing the contamination status of the Subaé estuarine system and/or biological responses resulting from pollutants.

It is well established that high concentrations of trace metals can have toxic effects on benthic organisms (e.g., Long et al., 1995; Gray, 1997). These effects generally involve alteration of species composition, reduction of richness and number of individuals, and may result in biodiversity losses (e.g., McGee et al., 1995). The aim of this study was to assess the current trace metal contamination of the sediments and the relationship between these contaminants, other environmental variables, and benthic macrofaunal assemblages.
Sediments and benthic samples were collected at several stations along the main channel of Subaé system in June 2004 (Fig. 1). Four soil samples were also collected in the vicinity of the lead smelter. Sediment samples (stations \#1-11), for geochemical analysis, were collected by SCUBA divers using a $5 \mathrm{~cm}$ diameter PVC core that was dragged, horizontally, for $2 \mathrm{~m}$ in surface sediments $(1-5 \mathrm{~cm})$. Once the sample was collected, the core was capped and the tube retrieved. Soils and river samples (\#12-18) were collected with plastic spoons. Samples were transferred to a precleaned LDPE container and kept frozen until analysis. All bottles and materials used for the collection and analysis were cleaned and immersed at least for $24 \mathrm{~h}$ in extran detergent solution and for a further $48 \mathrm{~h}$ in $10 \% \mathrm{HNO}_{3}$ solution, rinsed with ultra pure water, dried on a clean covered bench and stored in zip-lock bags before use.

Sediment samples were divided into two parts, the first used for the determination of particle-size distribution, and the second for chemical analyses. Before chemical analyses, sediments were wet sieved to separate the fraction smaller than $63 \mu \mathrm{m}$ that was used for trace metal analyses. A 'pseudo-total' digestion was performed using $250 \mathrm{mg}$ of sediments and $4 \mathrm{ml}$ of aqua regia in Teflon Parr bombs, for $16 \mathrm{~h}$ at a temperature of $120 \pm 5^{\circ} \mathrm{C}$. Additionally, an extraction using $20 \mathrm{ml}$ of $1 \mathrm{M} \mathrm{HCl}$, shaking for $12 \mathrm{~h}$ at room temperature was carried out. The latter extraction is reported to closely correlate with biological availability (Bryan and Langston, 1992). Sediment samples were digested/extracted in triplicates. Trace metals $(\mathrm{Co}, \mathrm{Cr}$, $\mathrm{Cu}, \mathrm{Pb}, \mathrm{Mn}, \mathrm{Ni}$ and $\mathrm{Zn}$ ), were determined by ICP OES (Varian, VISTA-PRO).

Blanks were included in each batch of analysis. The precision and accuracy of the analytical technique were 
Table 1

Detection limits, mean concentrations $\left(\mathrm{mg} \mathrm{kg}^{-1}\right)$ and standard deviations obtained for four replicates of the certified reference material MESS-2

\begin{tabular}{lccl}
\hline Metal & $\begin{array}{c}\text { Measured } \\
\left(\mathrm{mg} \mathrm{kg}^{-1}\right)\end{array}$ & $\begin{array}{l}\text { Certified } \\
\left(\mathrm{mg} \mathrm{kg}^{-1}\right)\end{array}$ & $\begin{array}{l}\text { Detection limits } \\
\left(\mathrm{mg} \mathrm{kg}^{-1}\right)\end{array}$ \\
\hline $\mathrm{Co}$ & $11.9 \pm 0.5$ & $13.8 \pm 1.4$ & 0.0042 \\
$\mathrm{Mn}$ & $300 \pm 2.5$ & $365 \pm 21$ & 0.3510 \\
$\mathrm{Cr}$ & $88.0 \pm 1.0$ & $106 \pm 8$ & 0.0039 \\
$\mathrm{Ni}$ & $36.8 \pm 1.7$ & $49.3 \pm 1.8$ & 0.0084 \\
$\mathrm{~Pb}$ & $12.8 \pm 1.1$ & $21.9 \pm 1.2$ & 0.0170 \\
$\mathrm{Zn}$ & $139 \pm 1.8$ & $172 \pm 16$ & 0.0011 \\
$\mathrm{Cu}$ & $28.2 \pm 1.5$ & $39.3 \pm 2$ & 0.0061 \\
$\mathrm{Cd}$ & $0.172 \pm 0.02$ & $0.24 \pm 0.01$ & 0.0007 \\
\hline
\end{tabular}

assessed by analysis of a Certified Reference Material, MESS-2 (National Research Council of Canada) and a Laboratory Reference Material SETS-1 with each batch of samples. The average and standard deviation of the results of MESS-2 analyses are given in Table 1. Results indicated good analytical precision, but incomplete digestion (58-87\%) for the aqua regia procedure. These results were not unexpected since the digestion procedure did not include hydrofluoric acid. All procedures including the sample collection and analyses were carefully undertaken in order to reduce potential contamination.

In order to examine the structure of the macrofaunal assemblages, two sites, $20 \mathrm{~m}$ apart, were randomly sampled at each station. At each site, four replicate cores $(10 \mathrm{~cm}$ diameter $\times 10 \mathrm{~cm}$ depth) were collected, by SCUBA divers, totaling 88 samples. Samples of sediment containing macrofauna were placed in plastic bags, immediately transported to land, sieved with a $0.5 \mathrm{~mm}$ mesh, preserved in $70 \%$ ethanol and maintained in a freezer for further sorting in the laboratory. In the laboratory, animals were counted and identified to intermediate taxonomic levels, usually family or morphotype. Considering that not only the biology and ecology, but also the taxonomic knowledge of the benthic fauna in the region is limited, the above procedure minimized the time taken to sort (James et al., 1995). Moreover, several studies have shown that identification to species level is not necessary for the detection of patterns of spatial and temporal variability in macrofaunal assemblages (e.g., Gray et al., 1990; Clarke and Warwick, 1994).

Multivariate analyses were performed with the samples collected in the estuary (i.e., \#1-11). A correlation-based principal component analyses (PCA) was used to investigate the distribution patterns of metal concentrations and grain size. For PCA, metal concentration data were log $(x+1)$ transformed. A non-metric multidimensional scaling (nMDS), based on Bray-Curtis dissimilarities of the fourth-root transformed macrofauna data, was used to investigate the biological spatial patterns. In this analysis, all the replicates of both sites were pooled $(n=8)$. The relationships between multivariate patterns of environmental variables and macrofaunal assemblages structure were examined using the BIOENV procedure (Clarke and Ainsworth, 1993; Clarke and Warwick, 1994). Analyses were performed using the software PRIMER V (Plymouth Marine Laboratorys UK). Data from station \#5 were not included in the nMDS and BIOENV analysis because the benthic samples, due to GPS failure, were collected in the intertidal zone.

Surface sediments were found to contain a wide range of trace metal concentrations (Table 2), from low $(\mathrm{Cd}$, $0.223 \mathrm{mg} \mathrm{kg}^{-1}$ ) to highly contaminated ( $\mathrm{Zn}, 877 \mathrm{mg} \mathrm{kg}^{-1}$ ). In soil samples (i.e., \#15-18), the concentrations of metals were even higher. Lead, for instance, showed concentrations up to $15,091 \mathrm{mg} \mathrm{kg}^{-1}$, while the $\mathrm{Zn}$ maximum concentration in soils was $2291 \mathrm{mg} \mathrm{kg}^{-1}$ (Table 2).

In general, the distribution patterns observed for trace metals extracted by aqua regia and $\mathrm{HCl}$ were the same, regardless of the metal concentrations in sediments, which varied over four orders of magnitude. Nickel and $\mathrm{Cr}$ concentrations were exceptions, and indicated some scatter in the distribution patterns obtained by the employed procedures. Among the studied elements, $\mathrm{Ni}$ and $\mathrm{Cr}$ showed the lowest extractability by $1 \mathrm{M} \mathrm{HCl}$, i.e., $30 \%$ and $10 \%$, respectively. Because $1 \mathrm{M} \mathrm{HCl}$ releases most non-residual metals, including some organic and sulfidic phases, but does not attack the silicate matrix, this result suggests that $\mathrm{Ni}$ and $\mathrm{Cr}$ were strongly associated with the lattice phase, indicating a low potential toxicity. On the other hand, on average, more than $80 \%$ of $\mathrm{Pb}$ and around $60 \%$ of $\mathrm{Cd}$, $\mathrm{Mn}$ and $\mathrm{Zn}$ were extracted using $1 \mathrm{M} \mathrm{HCl}$. In several stations the percentage of $\mathrm{Cd}$ extracted by $\mathrm{HCl}$ was as high as $90 \%$. Moreover, the high extractability of $\mathrm{Cd}, \mathrm{Zn}, \mathrm{Mn}$ and $\mathrm{Pb}$ with $\mathrm{HCl}$, a reducing agent, suggest that these metals were mainly solubilized by the dissolution of metallic oxides. Since metal bioavailability is related to metal solubility, it is to be expected that the latter elements were more bioavailable than $\mathrm{Ni}$ and $\mathrm{Cr}$.

Principal component analyses showed a gradient of contamination along the Subaé (Fig. 2). The first component (PC1) explained $44 \%$ of the variance and the second component (PC2) 23.9\%. The PC1 showed negative correlations with $\mathrm{CuT}(r=-0.31), \mathrm{CdT}(r=-0.30)$ and $\mathrm{ZnP}$ $(r=-0.26)$, and positive correlation with Silt $(r=0.26)$ and Clay $(r=0.26)$, while PC2 was negatively correlated with $\mathrm{PbP} \quad(r=-0.36), \quad \mathrm{PbT} \quad(r=-0.35)$ and $\mathrm{CrP}$ $(r=-0.33)$. Stations \#9 and \#11, which showed high concentrations of $\mathrm{Pb}, \mathrm{Zn}$ and $\mathrm{Cd}$, were relatively isolated from others in the PCA (Fig. 2).

The inactive $\mathrm{Pb}$ smelter appears to be an important fluvial and atmospheric source of metals, specially $\mathrm{Pb}, \mathrm{Zn}$ and Cd. Nevertheless, the proximity to the smelter, by itself, does not explain the high trace metal concentrations at stations \#9-11. Upstream of these stations, and hence closer to the smelter, the concentrations of metals in sediments were lower (Table 2).

During sampling, at low tide, salinity rose from 0.17 at \#11, reaching 27.5 at the estuarine mouth (\#1). It was observed that the low salinity region at stations \#9-11 showed the highest concentrations of suspended material (14.1-54.6 $\mathrm{mg} \mathrm{L}^{-1}$ ), and the lowest oxygen levels (3.46- 
Table 2

Mean and standard deviation $(n=3)$ of trace metal concentrations $\left(\mathrm{mg} \mathrm{kg}^{-1}\right)$ in sediment (\#1-14) and soil (\#15-18) samples and granulometry (\%) from Subaé River system, Brazil

\begin{tabular}{|c|c|c|c|c|c|c|c|c|c|c|c|c|c|c|c|c|c|c|c|c|}
\hline \multirow[t]{2}{*}{ Station } & \multicolumn{2}{|l|}{$\mathrm{Cd}$} & \multicolumn{2}{|l|}{$\mathrm{Co}$} & \multicolumn{2}{|l|}{$\mathrm{Cr}$} & \multicolumn{2}{|l|}{$\mathrm{Cu}$} & \multicolumn{2}{|l|}{$\mathrm{Mn}$} & \multicolumn{2}{|l|}{$\mathrm{Ni}$} & \multicolumn{2}{|l|}{$\mathrm{Pb}$} & \multicolumn{2}{|l|}{$\mathrm{Zn}$} & \multirow[t]{2}{*}{ Gravel } & \multirow[t]{2}{*}{ Sand } & \multirow[t]{2}{*}{ Silt } & \multirow[t]{2}{*}{ Clay } \\
\hline & $\mathrm{T}$ & $\mathrm{P}$ & $\mathrm{T}$ & $\mathrm{P}$ & $\mathrm{T}$ & $\mathrm{P}$ & $\mathrm{T}$ & $\mathrm{P}$ & $\mathrm{T}$ & $\mathrm{P}$ & $\mathrm{T}$ & $\mathrm{P}$ & $\mathrm{T}$ & $\mathrm{P}$ & $\mathrm{T}$ & $\mathrm{P}$ & & & & \\
\hline$\# 1$ & $\begin{array}{l}0.340 \\
(0.09)\end{array}$ & $\begin{array}{l}0.321 \\
(0.002)\end{array}$ & $\begin{array}{l}18.1 \\
(0.57)\end{array}$ & $\begin{array}{l}7.87 \\
(0.13)\end{array}$ & $\begin{array}{l}88.6 \\
(3.8)\end{array}$ & $\begin{array}{l}8.67 \\
(0.10)\end{array}$ & $\begin{array}{l}32.2 \\
(1.0)\end{array}$ & $\begin{array}{l}15.9 \\
(0.38)\end{array}$ & $\begin{array}{l}584 \\
(13)\end{array}$ & $\begin{array}{l}410 \\
(6.2)\end{array}$ & $\begin{array}{l}36.1 \\
(0.65)\end{array}$ & $\begin{array}{l}13.7 \\
(0.60)\end{array}$ & $\begin{array}{l}38.7 \\
(1.6)\end{array}$ & $\begin{array}{l}29.0 \\
(0.5)\end{array}$ & $\begin{array}{l}119 \\
(2.3)\end{array}$ & $\begin{array}{l}56.7 \\
(0.38)\end{array}$ & 0.024 & 26.2 & 29.5 & 44.3 \\
\hline$\# 2$ & $\begin{array}{l}0.350 \\
(0.10)\end{array}$ & $\begin{array}{l}0.336 \\
(0.01)\end{array}$ & $\begin{array}{l}16.6 \\
(0.16)\end{array}$ & $\begin{array}{l}7.18 \\
(0.02)\end{array}$ & $\begin{array}{l}88.7 \\
(0.71)\end{array}$ & $\begin{array}{l}9.02 \\
(0.09)\end{array}$ & $\begin{array}{l}33.2 \\
(0.35)\end{array}$ & $\begin{array}{l}17.2 \\
(0.23)\end{array}$ & $\begin{array}{l}375 \\
(6.0)\end{array}$ & $\begin{array}{l}244 \\
(1.8)\end{array}$ & $\begin{array}{l}40.5 \\
(0.78)\end{array}$ & $\begin{array}{l}14.4 \\
(0.26)\end{array}$ & $\begin{array}{l}35.5 \\
(1.0)\end{array}$ & $\begin{array}{l}26.6 \\
(0.14)\end{array}$ & $\begin{array}{l}134 \\
(1.1)\end{array}$ & $\begin{array}{l}73.3 \\
(1.1)\end{array}$ & 52.9 & 32.6 & 6.36 & 8.09 \\
\hline$\# 3$ & $\begin{array}{l}0.293 \\
(0.03)\end{array}$ & $\begin{array}{l}0.223 \\
(0.03)\end{array}$ & $\begin{array}{l}17.7 \\
(0.22)\end{array}$ & $\begin{array}{l}7.75 \\
(0.08)\end{array}$ & $\begin{array}{l}95.1 \\
(1.2)\end{array}$ & $\begin{array}{l}9.05 \\
(0.13)\end{array}$ & $\begin{array}{l}34.1 \\
(0.45)\end{array}$ & $\begin{array}{l}17.5 \\
(0.22)\end{array}$ & $\begin{array}{l}467 \\
(5.1)\end{array}$ & $\begin{array}{l}342 \\
(5.3)\end{array}$ & $\begin{array}{l}38.4 \\
(3.2)\end{array}$ & $\begin{array}{l}15.1 \\
(0.22)\end{array}$ & $\begin{array}{l}37.7 \\
(1.8)\end{array}$ & $\begin{array}{l}28.2 \\
(0.39)\end{array}$ & $\begin{array}{l}125 \\
(1.3)\end{array}$ & $\begin{array}{l}62.8 \\
(0.25)\end{array}$ & 0.041 & 1.66 & 38.0 & 60.3 \\
\hline$\# 4$ & $\begin{array}{l}0.767 \\
(0.01)\end{array}$ & $\begin{array}{l}0.700 \\
(0.02)\end{array}$ & $\begin{array}{l}18.7 \\
(0.22)\end{array}$ & $\begin{array}{l}7.51 \\
(0.05)\end{array}$ & $\begin{array}{l}99.9 \\
(0.72)\end{array}$ & $\begin{array}{l}8.81 \\
(0.15)\end{array}$ & $\begin{array}{l}41.3 \\
(0.85)\end{array}$ & $\begin{array}{l}21.8 \\
(0.22)\end{array}$ & $\begin{array}{l}277 \\
(2.6)\end{array}$ & $\begin{array}{l}151 \\
(2.5)\end{array}$ & $\begin{array}{l}37.9 \\
(1.0)\end{array}$ & $\begin{array}{l}15.4 \\
(0.95)\end{array}$ & $\begin{array}{l}42.7 \\
(0.03)\end{array}$ & $\begin{array}{l}33.2 \\
(0.50)\end{array}$ & $\begin{array}{l}131 \\
(0.62)\end{array}$ & $\begin{array}{l}66.2 \\
(0.21)\end{array}$ & 0.000 & 8.33 & 35.2 & 56.5 \\
\hline$\# 5$ & $\begin{array}{l}0.763 \\
(0.07)\end{array}$ & $\begin{array}{l}0.702 \\
(0.03)\end{array}$ & $\begin{array}{l}19.5 \\
(0.31)\end{array}$ & $\begin{array}{l}7.62 \\
(0.09)\end{array}$ & $\begin{array}{l}110 \\
(5.4)\end{array}$ & $\begin{array}{l}9.18 \\
(0.15)\end{array}$ & $\begin{array}{l}38.3 \\
(1.3)\end{array}$ & $\begin{array}{l}19.1 \\
(0.32)\end{array}$ & $\begin{array}{l}355 \\
(10)\end{array}$ & $\begin{array}{l}195 \\
(3.2)\end{array}$ & $\begin{array}{l}55.7 \\
(2.0)\end{array}$ & $\begin{array}{l}15.2 \\
(0.35)\end{array}$ & $\begin{array}{l}45.9 \\
(0.71)\end{array}$ & $\begin{array}{l}35.9 \\
(0.31)\end{array}$ & $\begin{array}{l}143 \\
(2.1)\end{array}$ & $\begin{array}{l}78.7 \\
(1.0)\end{array}$ & 1.14 & 84.3 & 6.63 & 8.0 \\
\hline$\# 6$ & $\begin{array}{l}1.41 \\
(0.05)\end{array}$ & $\begin{array}{l}0.569 \\
(0.01)\end{array}$ & $\begin{array}{l}19.2 \\
(0.34)\end{array}$ & $\begin{array}{l}6.45 \\
(0.08)\end{array}$ & $\begin{array}{l}95.8 \\
(2.6)\end{array}$ & $\begin{array}{l}7.51 \\
(0.02)\end{array}$ & $\begin{array}{l}44.9 \\
(2.4)\end{array}$ & $\begin{array}{l}20.4 \\
(0.09)\end{array}$ & $\begin{array}{l}416 \\
(16)\end{array}$ & $\begin{array}{l}268 \\
(2.2)\end{array}$ & $\begin{array}{l}40.4 \\
(4.3)\end{array}$ & $\begin{array}{l}16.1 \\
(0.38)\end{array}$ & $\begin{array}{l}45.8 \\
(1.9)\end{array}$ & $\begin{array}{l}36.0 \\
(0.25)\end{array}$ & $\begin{array}{l}196 \\
(0.83)\end{array}$ & $\begin{array}{l}125 \\
(1.7)\end{array}$ & 0.009 & 98.6 & 1.35 & 0.0 \\
\hline$\# 7$ & $\begin{array}{l}0.835 \\
(0.06)\end{array}$ & $\begin{array}{l}0.200 \\
(0.002)\end{array}$ & $\begin{array}{l}18.6 \\
(0.32)\end{array}$ & $\begin{array}{l}5.17 \\
(0.09)\end{array}$ & $\begin{array}{l}114 \\
(10)\end{array}$ & $\begin{array}{l}5.58 \\
(0.07)\end{array}$ & $\begin{array}{l}45.0 \\
(0.46)\end{array}$ & $\begin{array}{l}20.1 \\
(0.03)\end{array}$ & $\begin{array}{l}233 \\
(4.3)\end{array}$ & $\begin{array}{l}89.9 \\
(0.39)\end{array}$ & $\begin{array}{l}57.7 \\
(1.7)\end{array}$ & $\begin{array}{l}13.1 \\
(0.16)\end{array}$ & $\begin{array}{l}40.2 \\
(0.37)\end{array}$ & $\begin{array}{l}28.2 \\
(0.24)\end{array}$ & $\begin{array}{l}145 \\
(3.4)\end{array}$ & $\begin{array}{l}71.0 \\
(0.36)\end{array}$ & 1.40 & 97.1 & 1.54 & 0.0 \\
\hline$\# 8$ & $\begin{array}{l}2.06 \\
(0.08)\end{array}$ & $\begin{array}{l}1.04 \\
(0.01)\end{array}$ & $\begin{array}{l}23.6 \\
(0.30)\end{array}$ & $\begin{array}{l}6.16 \\
(0.05)\end{array}$ & $\begin{array}{l}102 \\
(9.2)\end{array}$ & $\begin{array}{l}5.66 \\
(0.12)\end{array}$ & $\begin{array}{l}49.9 \\
(0.60)\end{array}$ & $\begin{array}{l}15.4 \\
(0.08)\end{array}$ & $\begin{array}{l}298 \\
(3.2)\end{array}$ & $\begin{array}{l}101 \\
(0.09)\end{array}$ & $\begin{array}{l}53.4 \\
(7.0)\end{array}$ & $\begin{array}{l}14.7 \\
(0.16)\end{array}$ & $\begin{array}{l}41.7 \\
(1.6)\end{array}$ & $\begin{array}{l}29.4 \\
(0.06)\end{array}$ & $\begin{array}{l}171 \\
(3.2)\end{array}$ & $\begin{array}{l}98.8 \\
(0.93)\end{array}$ & 0.299 & 98.6 & 1.13 & 0.0 \\
\hline$\# 9$ & $\begin{array}{l}2.11 \\
(0.07)\end{array}$ & $\begin{array}{l}1.13 \\
(0.05)\end{array}$ & $\begin{array}{l}27.1 \\
(0.66)\end{array}$ & $\begin{array}{l}11.2 \\
(0.23)\end{array}$ & $\begin{array}{l}104 \\
(5.0)\end{array}$ & $\begin{array}{l}7.25 \\
(0.22)\end{array}$ & $\begin{array}{l}49.0 \\
(1.0)\end{array}$ & $\begin{array}{l}21.2 \\
(0.37)\end{array}$ & $\begin{array}{l}354 \\
(7.6)\end{array}$ & $\begin{array}{l}191 \\
(2.1)\end{array}$ & $\begin{array}{l}54.5 \\
(1.14)\end{array}$ & $\begin{array}{l}17.7 \\
(1.2)\end{array}$ & $\begin{array}{l}145 \\
(3.0)\end{array}$ & $\begin{array}{l}123 \\
(1.9)\end{array}$ & $\begin{array}{l}410 \\
(12)\end{array}$ & $\begin{array}{l}307 \\
(5.6)\end{array}$ & 0.323 & 97.9 & 1.74 & 0.0 \\
\hline$\# 10$ & $\begin{array}{l}0.850 \\
(0.09)\end{array}$ & $\begin{array}{l}0.525 \\
(0.03)\end{array}$ & $\begin{array}{l}37.5 \\
(1.22)\end{array}$ & $\begin{array}{l}15.7 \\
(0.18)\end{array}$ & $\begin{array}{l}113 \\
(8.4)\end{array}$ & $\begin{array}{l}7.80 \\
(0.17)\end{array}$ & $\begin{array}{l}43.9 \\
(0.97)\end{array}$ & $\begin{array}{l}10.4 \\
(0.17)\end{array}$ & $\begin{array}{l}323 \\
(3.4)\end{array}$ & $\begin{array}{l}119 \\
(1.34)\end{array}$ & $\begin{array}{l}72.5 \\
(5.14)\end{array}$ & $\begin{array}{l}23.6 \\
(1.3)\end{array}$ & $\begin{array}{l}17.4 \\
(1.3)\end{array}$ & $\begin{array}{l}10.9 \\
(0.11)\end{array}$ & $\begin{array}{l}151 \\
(7.04)\end{array}$ & $\begin{array}{l}85.8 \\
(0.67)\end{array}$ & 3.23 & 91.8 & 4.93 & 0.0 \\
\hline$\# 11$ & $\begin{array}{l}1.71 \\
(0.06)\end{array}$ & $\begin{array}{l}0.853 \\
(0.04)\end{array}$ & $\begin{array}{l}32.3 \\
(0.22)\end{array}$ & $\begin{array}{l}15.7 \\
(0.29)\end{array}$ & $\begin{array}{l}97.9 \\
(2.1)\end{array}$ & $\begin{array}{l}9.45 \\
(0.34)\end{array}$ & $\begin{array}{l}49.9 \\
(0.40)\end{array}$ & $\begin{array}{l}21.5 \\
(0.35)\end{array}$ & $\begin{array}{l}382 \\
(0.22)\end{array}$ & $\begin{array}{l}209 \\
(1.9)\end{array}$ & $\begin{array}{l}44.9 \\
(0.76)\end{array}$ & $\begin{array}{l}20.5 \\
(1.3)\end{array}$ & $\begin{array}{l}363 \\
(2.6)\end{array}$ & $\begin{array}{l}325 \\
(2.5)\end{array}$ & $\begin{array}{l}877 \\
(11)\end{array}$ & $\begin{array}{l}667 \\
(6.5)\end{array}$ & 0.961 & 98.6 & 0.448 & 0.0 \\
\hline$\# 12$ & $\begin{array}{l}1.56 \\
(0.13)\end{array}$ & $\begin{array}{l}0.414 \\
(0.02)\end{array}$ & $\begin{array}{l}32.5 \\
(0.33)\end{array}$ & $\begin{array}{l}8.62 \\
(0.11)\end{array}$ & $\begin{array}{l}105 \\
(6.2)\end{array}$ & $\begin{array}{l}5.07 \\
(0.28)\end{array}$ & $\begin{array}{l}47.6 \\
(5.3)\end{array}$ & $\begin{array}{l}18.6 \\
(0.37)\end{array}$ & $\begin{array}{l}936 \\
(1.7)\end{array}$ & $\begin{array}{l}776 \\
(6.1)\end{array}$ & $\begin{array}{l}57.5 \\
(1.6)\end{array}$ & $\begin{array}{l}11.8 \\
(1.6)\end{array}$ & $\begin{array}{l}175 \\
(1.4)\end{array}$ & $\begin{array}{l}53.7 \\
(0.6)\end{array}$ & $\begin{array}{l}479 \\
(18)\end{array}$ & $\begin{array}{l}115 \\
(0.55)\end{array}$ & 0.009 & 86.7 & 0.897 & 12.4 \\
\hline$\# 13$ & $\begin{array}{l}0.447 \\
(0.11)\end{array}$ & $\begin{array}{l}0.146 \\
(0.01)\end{array}$ & $\begin{array}{l}25.5 \\
(0.31)\end{array}$ & $\begin{array}{l}11.3 \\
(0.03)\end{array}$ & $\begin{array}{l}145 \\
(1.6)\end{array}$ & $\begin{array}{l}4.86 \\
(0.12)\end{array}$ & $\begin{array}{l}38.5 \\
(5.5)\end{array}$ & $\begin{array}{l}13.6 \\
(0.02)\end{array}$ & $\begin{array}{l}890 \\
(6.0)\end{array}$ & $\begin{array}{l}727 \\
(5.4)\end{array}$ & $\begin{array}{l}70.1 \\
(1.33)\end{array}$ & $\begin{array}{l}13.4 \\
(0.29)\end{array}$ & $\begin{array}{l}39.2 \\
(2.7)\end{array}$ & $\begin{array}{l}39.3 \\
(0.40)\end{array}$ & $\begin{array}{l}118 \\
(7.8)\end{array}$ & $\begin{array}{l}52.1 \\
(0.65)\end{array}$ & 0.621 & 72.0 & 3.17 & 24.3 \\
\hline \#14 & $\begin{array}{l}0.386 \\
(0.03)\end{array}$ & $\begin{array}{l}0.026 \\
(0.008)\end{array}$ & $\begin{array}{l}24.4 \\
(0.02)\end{array}$ & $\begin{array}{l}10.0 \\
(0.13)\end{array}$ & $\begin{array}{l}80.8 \\
(9.0)\end{array}$ & $\begin{array}{l}4.45 \\
(0.22)\end{array}$ & $\begin{array}{l}39.7 \\
(3.0)\end{array}$ & $\begin{array}{l}15.0 \\
(0.31)\end{array}$ & $\begin{array}{l}760 \\
(5.2)\end{array}$ & $\begin{array}{l}598 \\
(2.4)\end{array}$ & $\begin{array}{l}36.2 \\
(0.73)\end{array}$ & $\begin{array}{l}12.2 \\
(1.65)\end{array}$ & $\begin{array}{l}31.7 \\
(0.8)\end{array}$ & $\begin{array}{l}30.6 \\
(0.13)\end{array}$ & $\begin{array}{l}113 \\
(3.1)\end{array}$ & $\begin{array}{l}44.1 \\
(1.12)\end{array}$ & 0.217 & 89.5 & 1.05 & 9.20 \\
\hline$\# 15$ & $\begin{array}{l}70.3 \\
(0.42)\end{array}$ & $\begin{array}{l}59.3 \\
(1.1)\end{array}$ & $\begin{array}{l}21.4 \\
(0.11)\end{array}$ & $\begin{array}{l}9.45 \\
(0.08)\end{array}$ & $\begin{array}{l}84.5 \\
(3.0)\end{array}$ & $\begin{array}{l}6.49 \\
(0.11)\end{array}$ & $\begin{array}{l}281 \\
(1.9)\end{array}$ & $\begin{array}{l}204 \\
(6.9)\end{array}$ & $\begin{array}{l}556 \\
(5.9)\end{array}$ & $\begin{array}{l}439 \\
(2.3)\end{array}$ & $\begin{array}{l}40.6 \\
(2.1)\end{array}$ & $\begin{array}{l}22.9 \\
(0.84)\end{array}$ & $\begin{array}{l}4899 \\
(234)\end{array}$ & $\begin{array}{l}3660 \\
(33)\end{array}$ & $\begin{array}{l}1406 \\
(21)\end{array}$ & $\begin{array}{l}958 \\
(3.37)\end{array}$ & 5.63 & 17.5 & 7.09 & 69.8 \\
\hline$\# 16$ & $\begin{array}{l}115 \\
(3.4)\end{array}$ & $\begin{array}{l}91.1 \\
(1.3)\end{array}$ & $\begin{array}{l}19.3 \\
(0.5)\end{array}$ & $\begin{array}{l}7.40 \\
(0.25)\end{array}$ & $\begin{array}{l}104.1 \\
(6.5)\end{array}$ & $\begin{array}{l}6.74 \\
(0.30)\end{array}$ & $\begin{array}{l}552 \\
(13)\end{array}$ & $\begin{array}{l}393 \\
(8.55)\end{array}$ & $\begin{array}{l}630 \\
(18)\end{array}$ & $\begin{array}{l}484 \\
(8.9)\end{array}$ & $\begin{array}{l}55.7 \\
(6.5)\end{array}$ & $\begin{array}{l}21.4 \\
(1.7)\end{array}$ & $\begin{array}{l}15091 \\
(743)\end{array}$ & $\begin{array}{l}9421 \\
(20)\end{array}$ & $\begin{array}{l}2291 \\
(70)\end{array}$ & $\begin{array}{l}1204 \\
(10)\end{array}$ & 5.76 & 44.7 & 4.55 & 45.0 \\
\hline \#17 & $\begin{array}{l}2.15 \\
(0.02)\end{array}$ & $\begin{array}{l}1.06 \\
(0.04)\end{array}$ & $\begin{array}{l}17.6 \\
(0.21)\end{array}$ & $\begin{array}{l}7.28 \\
(0.30)\end{array}$ & $\begin{array}{l}72.3 \\
(0.5)\end{array}$ & $\begin{array}{l}6.10 \\
(0.08)\end{array}$ & $\begin{array}{l}45.2 \\
(0.6)\end{array}$ & $\begin{array}{l}18.2 \\
(0.27)\end{array}$ & $\begin{array}{l}578 \\
(6.4)\end{array}$ & $\begin{array}{l}472 \\
(14)\end{array}$ & $\begin{array}{l}32.1 \\
(0.5)\end{array}$ & $\begin{array}{l}20.2 \\
(0.35)\end{array}$ & $\begin{array}{l}153 \\
(2.5)\end{array}$ & $\begin{array}{l}130 \\
(2.0)\end{array}$ & $\begin{array}{l}115 \\
(0.6)\end{array}$ & $\begin{array}{l}59.2 \\
(1.2)\end{array}$ & 5.16 & 69.1 & 2.46 & 23.3 \\
\hline$\# 18$ & $\begin{array}{l}5.85 \\
(0.11)\end{array}$ & $\begin{array}{l}5.21 \\
(0.03)\end{array}$ & $\begin{array}{l}19.5 \\
(0.32)\end{array}$ & $\begin{array}{l}9.85 \\
(0.4)\end{array}$ & $\begin{array}{l}97.5 \\
(22.5)\end{array}$ & $\begin{array}{l}7.96 \\
(0.09)\end{array}$ & $\begin{array}{l}52.2 \\
(6.1)\end{array}$ & $\begin{array}{l}21.1 \\
(0.22)\end{array}$ & $\begin{array}{l}510 \\
(8.5)\end{array}$ & $\begin{array}{l}395 \\
(9.2)\end{array}$ & $\begin{array}{l}41.4 \\
(1.5)\end{array}$ & $\begin{array}{l}22.6 \\
(0.81)\end{array}$ & $\begin{array}{l}164 \\
(1.1)\end{array}$ & $\begin{array}{l}145 \\
(4.2)\end{array}$ & $\begin{array}{l}239 \\
(9.1)\end{array}$ & $\begin{array}{l}172 \\
(1.8)\end{array}$ & 4.50 & 74.8 & 2.39 & 18.3 \\
\hline
\end{tabular}

Pseudo-total fraction $=\mathrm{T}$, and $\mathrm{HCl}$ extracts $=\mathrm{P}$. 


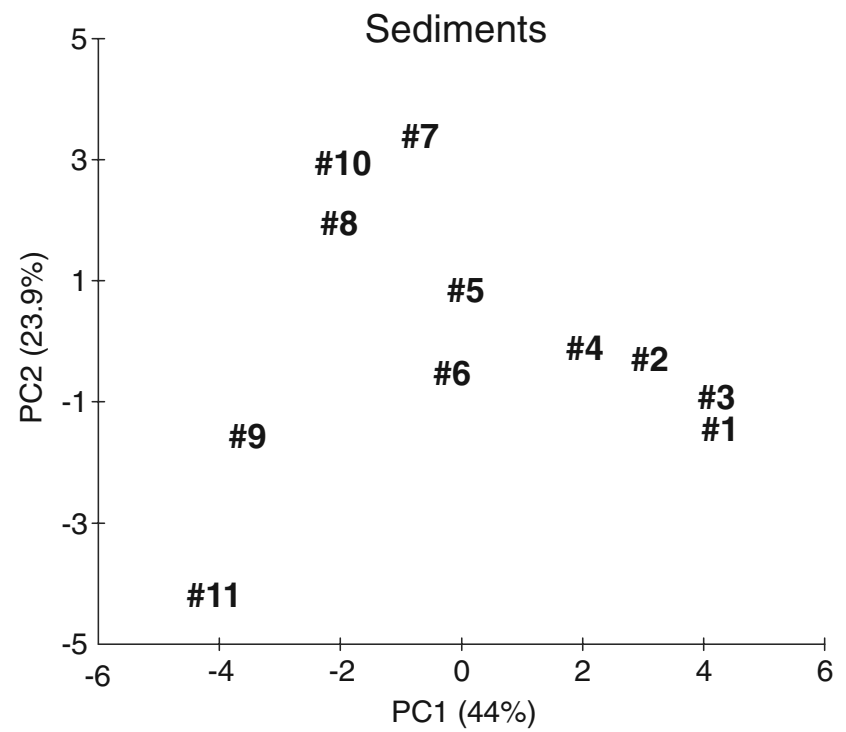

Fig. 2. Principal component analyses performed with environmental variables at each station of the Subaé estuary, Brazil.

$4.66 \mathrm{mg} \mathrm{L}^{-1}$ ). The high concentrations of metals encountered in this region are, therefore, more likely to be associated with the physico-chemistry of the waters rather than proximity to the $\mathrm{Pb}$ smelter. The presence of electrolytes at the freshwater/seawater interface seems to be causing flocculation of colloids and precipitation of manganese and iron oxides, exerting scavenging effects on susceptible metals that accumulate in the sediments. Nevertheless, two fishing villages also seem to have an impact in metal concentrations and physico-chemistry of the waters in this area, since they release untreated sewage directly into the estuary.

The nMDS (Fig. 3) also showed that stations \#9-11 were dissimilar from other stations, corroborating the environmental data (Fig. 2). Invertebrates at station \#2 showed relatively high diversity and abundance compared to other stations, which can be accounted for by the greater proportion of gravel, composed by shell fragments, increasing sediment heterogeneity (Table 2, Fig. 3). Furthermore, coarse sediments have greater permeability and porosity than fine sediments, which allows easy water circulation and therefore low accumulation of contaminants.

The best combination of environmental variables ( CdP, CoT, CoP, NiT, Gravel and Silt) moderately explained the structure of benthic assemblage patterns (BIOENV, $\rho=0.63$ ), indicating that there were other important factors (perhaps other contaminants such as polycyclic aromatic hydrocarbons, and nutrients) that were not included in the analysis. Nevertheless, results suggested that the structure of the benthic macrofaunal assemblages, at all stations, was negatively affected by high concentrations of Co. This trace metal, alone, was reasonably correlated with the structure of the benthic macrofauna (BIOENV, CoT: $\rho=0.58$, CoP: $\rho=0.44$ ).

Both the biological and the environmental data showed a significant gradient along the estuary (RELATE,

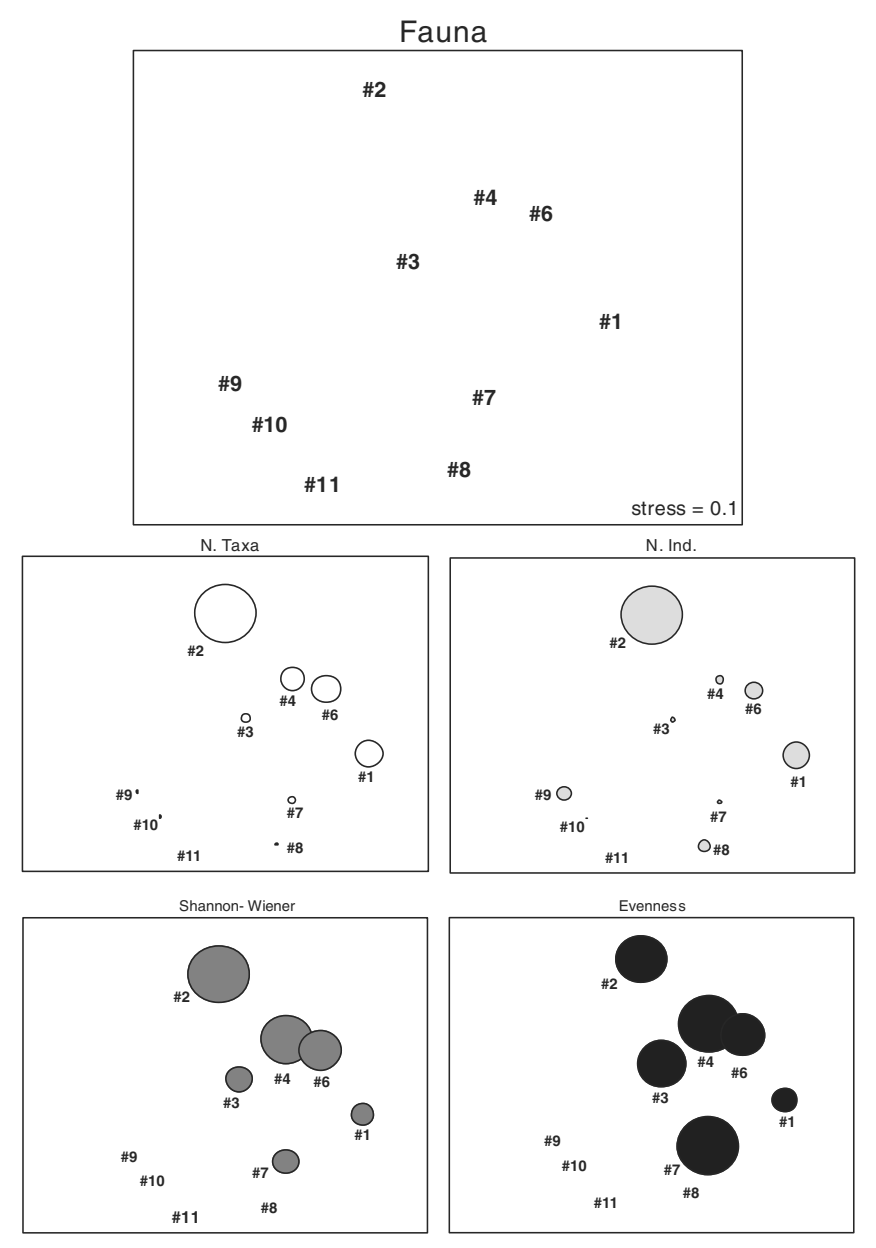

Fig. 3. Non-metric multidimensional scaling performed with the macrofaunal assemblages data at each of the subtidal stations of the Subaé. Different circles sizes are proportional to the original values of the total number of taxa (N. Taxa) and individuals (N. Ind.), diversity index (Shannon-Wiener, $\log$ e) and Pielous's Evenness (Evenness) at each station.

$\rho>0.65, p<0.01$, ) and roughly matched spatial patterns in each ordination (nMDS and PCA). Nevertheless, conversely to initial expectations, it seems that macrofaunal assemblages responded more strongly to the pseudo-total metal concentrations than the metals extracted by $\mathrm{HCl}$, i.e., the more bioavailable fraction. However, this relationship requires further testing.

In order to estimate the biological significance of trace metal levels in the sediments of the Subae estuary, the concentrations obtained in this study were compared to sediment quality data (NOAA, 1999). For all stations, the concentrations of $\mathrm{Co}$ and $\mathrm{Mn}$ were above Apparent Effect Thresholds (AET). The high background level for $\mathrm{Mn}$ in BTS $\left(344 \pm 105 \mathrm{mg} \mathrm{kg}^{-1}\right)$ and elevated concentrations distributed all along this bay, nevertheless, suggest that the elevated values are due to the lithology of the basin (CRA, 2004). Zinc concentrations at stations \#9, \#11 and \#12 were also above AET levels. Concentrations above AET represent conditions at which adverse biological impacts would always be expected. In more than $40 \%$ of 
stations the concentrations of Ni were above Effects Range Median (ERM). In several stations concentrations of $\mathrm{Cd}$, $\mathrm{Cr}$ and $\mathrm{Cu}$ were above Effects Range-Low (ERL), a level above which toxicity may begin to be observed in sensitive species.

In summary, the results showed that the potentially most adverse environmental conditions for biota occurred at stations \#8, \#9, \#10 and \#11. The fact that the benthic macrofauna in these stations also presented the smallest number of taxa and individuals, reinforced the probability of harmful biological effects being present. These results indicated that the inactive $\mathrm{Pb}$ smelter is an important contemporaneous source of trace metals for the Subaé system. The contaminants from the disposal site still present high mobility, increasing the potential for continuous environmental impact, not only due to surface run-off but also the movement of ground water.

\section{Acknowledgements}

We thank G. Lessa, A.B.A. Góes, for field and lab assistance. We also thank CAPES and $\mathrm{CNPq}$ for funding support. Hatje and Barros were financially assisted by fellowships from CAPES and CNPq, respectively. FUNDIPESCA assisted with field work.

\section{References}

Bryan, G.W., Langston, W.J., 1992. Bioavailability, accumulation and effects of heavy metals in sediments with special reference to United Kingdom estuaries, a review. Environmental Pollution 76, 89-131.

Carvalho, F.M., Tavares, T.M., Souza, S.P., Linhares, P., 1984. Lead and cadmium concentrations in the hair of fishermen from Subae basin, Brazil. Environmental Research 33, 300-306.

Carvalho, F.M., Silvany-Neto, A.M., Lima, M.E.C., Tavares, T.M., Waldron, H.A., Souza, S.P., Linhares, P., 1985. Lead poisoning among children from Santo Amaro, Bahia, Brazil. Bulletin Pan American Health Organization 19, 165-175.
Carvalho, F.M., Silvany-Neto, A.M., Melo, A.M.C., Chaves, M.E.C., Brandão, A.M., Tavares, T.M., 1989. Cadmium in hair of children living near smelter in Brazil. The Science of the Total Environment 84, 119-128.

Carvalho, F.M., Silvany-Neto, A.M., Tavares, T.M., Costa, A.C.A., Reis, M.A., 2003. Chumbo no sangue de crianças e passivo ambiental de uma fundição de chumbo no Brasil. Pan American Journal of Public Health 13, 19-24.

Clarke, K.R., Ainsworth, M., 1993. A method of linking multivariate community structure to environmental variables. Marine Ecology Progress Series 92, 205-219.

Clarke, K.R., Warwick, R.M., 1994. Change in Marine Communities: An Approach To Statistical Analysis And Interpretation. Natural Environment Research Council/Plymouth Marine Laboratory, UK, Plymouth.

CRA, 2004. Diagnóstico ambiental do grau de contaminação da baía de todos os santos por metais pesados e hidrocarbonetos. CRA, Salvador.

Gray, J.S., 1997. Marine biodiversity: patterns, threats and conservation needs. Biodiversity and Conservation 6, 153-175.

Gray, J.S., Clarke, K.R., Warwick, R.M., Hobbs, G., 1990. Detection of initial effects of pollution on marine benthos: an example from the Ekofisk and Eldfisk oilfields, North Sea. Marine Ecology Progress Series 66, 285-299.

James, R.J., Smith, M.P.L., Fairweather, P.G., 1995. Sieve mesh size and taxonomic resolution needed to describe natural spatial variation of marine macrofauna. Marine Ecology Progress Series 118, 187-198.

Long, E.R., McDonald, D.D., Smith, S.L., Calder, F.D., 1995. Incidence of adverse biological effects with ranges of chemical concentrations in marine and estuarine sediments. Environmental Management 19, 81-97.

McGee, B.L., Schlekat, C.E., Boward, D.M., Wade, T.L., 1995. Sediment contamination and biological effects in a Chesapeake Bay marina. Ecotoxicology 4, 39-59.

NOAA, 1999. Screening quick reference tables. NOAA HAZMAT Report 99-1, Coastal Protection and Restoration Division, National Oceanic and Atmospheric Administration, Seattle, WA.

Reis, J.O.N. 1975. Determinação polarográfica de $\mathrm{Pb}^{2+}$ and $\mathrm{Cd}^{2+}$ em águas do Rio Subaé, Santo Amaro, Bahia. Master thesis, Universidade Federal da Bahia, Brazil, p. 81.

Silvany-Neto, A.M., Carvalho, F.M., Chaves, M.E.C., Brandão, A.M., Tavares, T.M., 1989. Repeated surveillance of lead poisoning among children. The Science of the Total Environment 78, 178-186.

Tavares, T.M. Programa de monitoramento dos ecossistemas ao norte da baía de todos os santos, 1996 Relatório Final, Universidade Federal da Bahia Salvador. 